PSICOLOGIA, SAÚDE \& DOENÇAS, 2021, 22(1), 182-194

ISSN - 2182-8407

Sociedade Portuguesa de Psicologia da Saúde - SPPS - www.sp-ps.pt

DOI: http://dx.doi.org/10.15309/21psd220116

\title{
RELIGIOSIDADE/ ESPIRITUALIDADE, BEM-ESTAR E SATISFAÇÃO CONJUGAL EM CASAMENTOS DE LONGA DURAÇÃO
}

\author{
Suzel Goulart ${ }^{1}$, Heloísa Ferreira ${ }^{2,3}$, Clarisse Mosmann ${ }^{4,5}, \&$ Fabio Scorsolini-Comin ${ }^{6}$ \\ ${ }^{1}$ Universidade Federal do Triângulo Mineiro, Uberaba-MG, Brasil, suzel_goulart@hotmail.com \\ ${ }^{2}$ Universidade Federal de São Carlos, São Paulo, Brasil, helogf@gmail.com \\ ${ }^{3}$ Universidade do Estado do Rio de Janeiro, Rio de Janeiro, Brasil. \\ ${ }^{4}$ Pontifícia Universidade Católica do Rio Grande do Sul, Rio Grande do Sul, Brasil, clarissemosmann@gmail.com \\ ${ }^{5}$ Universidade do Vale do Rio dos Sinos, São Leopoldo - Rio Grande do Sul, Brasil. \\ ${ }^{6}$ Universidade de São Paulo, Ribeirão Preto - São Paulo, Brasil, fabio.scorsolini@usp.br
}

RESUMO: A literatura científica tem sinalizado que a religiosidade/espiritualidade (R/E) é um componente importante no relacionamento conjugal, podendo estar associado à sua manutenção ao longo do tempo, bem como a indicadores de bem-estar. $\mathrm{O}$ objetivo deste estudo foi avaliar as associações entre as medidas de bem-estar espiritual, bem-estar subjetivo, coping religioso-espiritual e satisfação conjugal em casais longevos. Participaram 111 brasileiros casados havia, no mínimo, 15 anos $(M=26,05 ; D P=7,47)$, que responderam à Escala de Satisfação Conjugal-GRIMS, Escala de Bem-Estar Espiritual, Escala de Satisfação de Vida, Escala de Afetos Positivos e Negativos-PANAS C-8, Escala de Coping Religioso-Espiritual e Questionário Sociodemográfico. Foram realizadas análises descritivas e correlacionais. A interpretação baseou-se na Psicologia Positiva. Pode-se concluir que pessoas com maiores níveis de $\mathrm{R} / \mathrm{E}$ e bem-estar tendem a ter menos problemas conjugais e a relatar mais satisfação com seus casamentos. A partir dos resultados, evidenciou-se que a $\mathrm{R} / \mathrm{E}$ pode ser um componente importante na manutenção do casamento em uniões longevas.

Palavras-Chave: relações conjugais, religiosidade, espiritualidade.

\section{RELIGIOSITY/ SPIRITUALITY, WELL-BEING AND MARITAL SATISFACTION IN LONG-TERM MARRIAGES COPING}

\begin{abstract}
The scientific literature has signaled that religiosity/spirituality $(\mathrm{R} / \mathrm{S})$ is an important component in the marital relationship, and may be associated with its maintenance over time, as well as with well-being indicators. The aim of this study was to evaluate how to measure the relation between spiritual well-being, well-being, religious-spiritual coping and marital satisfaction in long-term couples. The sample was collected from Brazilian married people $(n=111)$, at least 15 years old $(M=26.05, S D=$ 7.47). The instruments were the GRIMS Conjugal Satisfaction Scale, Spiritual WellBeing Scale, Life Satisfaction Scale, Positive and Negative Affective Scales - PANAS C8, Religious-Spiritual Scale of Scale, and Sociodemographic Questionnaire. The
\end{abstract}

\footnotetext{
Departamento de Enfermagem Psiquiátrica e Ciências Humanas, Escola de Enfermagem de Ribeirão Preto, Universidade de São Paulo, Avenida Bandeirantes, 3900, Monte Alegre, Ribeirão Preto - São Paulo, Brasil, CEP: 14040-902, email: fabio.scorsolini@usp.br
} 
descriptive and calculated measures were performed between the measures of the continuous variables of the factor of the instruments by the Spearman coefficient. The interpretation was based on Positive Psychology. People with higher levels of R/S and well-being tend to present more conjugated and more satisfactory problems with their marriages. From the results, it became evident that $\mathrm{R} / \mathrm{S}$ can be an important component in maintaining marriage in long-lived unions.

Keywords: marital relations, religiosity, spirituality.

Recebido em 31 de março de 2020/ Aceite em 16 de março de 2021

O interesse e a busca pela compreensão da religiosidade e da espiritualidade estiveram sempre presentes na história humana (Nadal et al., 2018). Apesar das diferenças epistemológicas entre os dois termos, neste estudo empregaremos a nomenclatura combinada, ou seja, religiosidade/espiritualidade $(\mathrm{R} / \mathrm{E})$, em consonância com as investigações no campo da saúde que priorizam as ressonâncias/desfechos dessa dimensão e as suas relações com o bem-estar (Cunha et al., 2020; Cunha \& Scorsolini-Comin, 2020).

No campo da conjugalidade de longa duração, ou seja, de casamentos com mais de 15 anos, alguns estudos têm investigado de que modo a $\mathrm{R} / \mathrm{E}$ está presente na vida dos casais, tanto em termos da compreensão das variáveis possivelmente associadas à escolha pelo matrimônio, da manutenção do casamento, como de busca por um sentido de vida que pode ser individual ou compartilhado pela díade (Alves-Silva et al., 2017; Goulart et al., 2019). A pesquisa de Mullins (2016), conduzida nos Estados Unidos, revelou que os casais relataram quatro contextos sociais que deram significados sagrados ao seu casamento: oração, serviço, adoração e comunhão. Esses contextos ampliaram o significado do sagrado dentro do repertório cultural e religioso, reforçando a percepção dos casais mais velhos de seus casamentos como sagrados, por meio do reconhecimento formal e público da longevidade da união, e como bênção de Deus, promovendo significados positivos. Efeitos negativos da religião sobre o casamento foram evidenciados pelos casais quando as experiências vividas da religião entravam em conflito com suas expectativas culturais para casamentos duradouros.

A pesquisa realizada por Estrada (2009), nos Estados Unidos, sobre amor e satisfação conjugal em casamentos de longa duração, concluiu que religião, felicidade, perdão e satisfação se mostram aspectos fundamentais nos relacionamentos dos participantes, sendo identificados pelos casais como "a chave" para um casamento duradouro. Norgren et al. (2004), em um dos estudos pioneiros sobre o tema no contexto brasileiro, destacaram que questões relacionadas à $\mathrm{R} / \mathrm{E}$ estão presentes nesses relacionamentos e apontam que o nível de satisfação conjugal é maior quando esses casais são praticantes de um grupo religioso, e que maiores níveis de $\mathrm{R} / \mathrm{E}$ parecem diminuir a possiblidade de divórcio e facilitar o funcionamento marital.

Assim como a R/E, o bem-estar subjetivo está presente em diversos estudos que abrangem a conjugalidade, sendo composto pelas dimensões afetos positivos, afetos negativos e satisfação com a vida (Scorsolini-Comin et al., 2015; Scorsolini-Comin \& Santos, 2011). Dentre esses estudos, ressalta-se a pesquisa realizada por Scorsolini-Comin et al. (2012) sobre a expressão do afeto e bemestar subjetivo em pessoas casadas. Nesta, destacou-se que, quanto mais a pessoa se sente feliz, mais tende a expressar sua afetividade quando está com o(a) parceiro(a).

Nesse sentido, destaca-se que a satisfação conjugal é resultado das expectativas que os cônjuges têm em comparação com a realidade vivenciada no casamento (Mosmann et al., 2006; Rainal \& Maity, 2018). Pressupondo que seja influenciada por diversas variáveis como, por exemplo, características da personalidade, valores, atitudes, sexo e nível sociodemográfico, a satisfação conjugal é considerada um fenômeno complexo. Tal aspecto ocorre em função do casamento passar por diversas transformações ao longo do ciclo de vida familiar, repercutindo no nível de satisfação que também varia com o decorrer dos anos de convívio conjugal (Norgren et al., 2004). 


\section{CASAMENTOS DE LONGA DURAÇÃO}

Diante do exposto, percebe-se que a $\mathrm{R} / \mathrm{E}$ está presente em alguns estudos sobre conjugalidade (Garcia \& Maciel, 2008; Marks, 2005), sendo mencionada como uma variável de destaque, mas poucas pesquisas analisam, de fato, a influência da $\mathrm{R} / \mathrm{E}$ em casamentos de longa duração (Grizólio et al., 2020), bem como outras variáveis associadas a essa expressão, como o bem-estar subjetivo. Desse modo, este estudo propõe-se a preencher duas importantes lacunas, sobretudo na literatura científica nacional. A primeira delas se refere ao estudo da conjugalidade de longa duração, compreendendo melhor esse fenômeno em um cenário cada vez mais atingido pelas altas taxas de divórcio e de fragilização dos vínculos amorosos (Alves-Silva et al., 2017; Campos et al., 2017; Costa et al., 2015; Costa \& Mosmann, 2015; Grizólio et al., 2020; Silva et al., 2017). Ainda, ressalta-se a possibilidade de ampliar o estudo sobre a $\mathrm{R} / \mathrm{E}$ em casamentos longevos.

A partir desse panorama, o objetivo do estudo foi avaliar as associações entre as medidas de bemestar espiritual, bem-estar subjetivo, coping religioso-espiritual e da satisfação conjugal em casais longevos. Como a literatura científica tem apontado que pessoas com maior satisfação no casamento tendem a manifestar maior satisfação com a R/E, e com maiores índices de bem-estar, a hipótese do estudo é que a $\mathrm{R} / \mathrm{E}$ nos casamentos de longa duração está positiva e significativamente associada à satisfação conjugal.

\section{MÉTODO}

\section{Tipo de estudo e aspectos éticos}

Trata-se de um estudo de corte transversal, de caráter correlacional, amparado na metodologia quantitativa de pesquisa, aprovado pelo Comitê de Ética em Pesquisa da Universidade Federal do Triângulo Mineiro (CAAE 775014174.0000.5154 e Parecer 2.376.725).

\section{Participantes}

Em relação aos critérios de inclusão/exclusão, foram definidos para inclusão de participantes no estudo: estar consensualmente casado há, no mínimo, 15 anos, de ambos os sexos, e não estar em processo de separação conjugal. O tempo mínimo de 15 anos foi estabelecido em função das estimativas do IBGE acerca da duração média dos casamentos no Brasil. Não houve restrições com relação à duração máxima para os casamentos, quantidade de filhos, aspectos socioeconômicos, grau de instrução, denominação religiosa, ter ou não uma religião, ou frequência mínima de participação em ritos religiosos. Dessa forma, a amostra total foi do tipo não probabilística, composta por critérios de conveniência. Participaram deste estudo 111 indivíduos com idades variando entre 37 a 67 anos $(M=49,63 ; D P=7,47)$, sendo $85,6 \%$ do sexo feminino e $14,4 \%$ do sexo masculino. Em relação ao tempo de união, a média foi de 26,05 anos $(D P=7,50)$. Verificou-se que $94,6 \%$ da mostra possuem filhos, considerando a média de 2,09 filhos $(D P=0,78)$ por participante. A renda familiar variou de forma heterogênea, sendo que dentre os sujeitos $4,5 \%$ ganhavam um salário mínimo, $18,9 \%$ recebiam de dois a três salários mínimos, $17,1 \%$ até cinco salários mínimos, 24,3\% ganhavam entre cinco a 10 salários mínimos, 30,6\% mais de 10 salários mínimos e 4,5\% ganhavam mais de 20 salários mínimos. Em termos de grau de instrução dos participantes, a maioria (43,2\%) possuía pós-graduação, o que se observou tanto entre os homens quanto entre as mulheres. Acerca das profissões/ocupações, $82 \%$ da amostra exercia alguma atividade laboral, com destaque para a Psicologia e também para a docência. Dessa forma, pode-se dizer que a maioria dos participantes possuía nível superior de instrução e exerciam atividade remunerada, com vínculo formal de trabalho.

\section{Instrumentos}


Escala de Satisfação Conjugal - GRIMS (Falcke, 2003): composta por 28 itens, mede a qualidade do relacionamento conjugal através das dimensões satisfação, comunicação, interesses compartilhados, confiança e respeito. Na pontuação da escala, verifica-se que quanto menor a pontuação obtida maior a qualidade conjugal e, quanto maior a pontuação, mais severos são os problemas no relacionamento conjugal (traduzida e adaptada à língua portuguesa por Falcke, 2003). Na presente amostra $(N=111)$, o alfa de Cronbach total da foi de 0,90 , o que define um nível de confiabilidade ideal da escala (Pasquali, 2001).

Escala de Bem-estar Espiritual (EBE) (Marques et al., 2009): Instrumento de referência para estudo e mensuração da espiritualidade, é subdividido em duas subescalas (10 itens cada) uma de Bem-estar Religioso (BER) e os outros 10 à mensuração do Bem-estar Existencial. Os itens de BER contêm uma referência a Deus, e os de Bem-estar Espiritual referem-se a uma sensação de encontro de algo significativo na vida. Na presente amostra, o alfa de Cronbach obtido também foi de 0,92 para BER, 0,77 para Bem-estar Espiritual e de 0,90 para a escala geral, sendo observada boa confiabilidade tanto na escala geral quanto em todas as subescalas.

Escala de Satisfação de Vida: Essa escala tem como objetivo acessar a satisfação de vida global dos participantes e consiste numa versão adaptada por Koller e Hutz (1996) da Satisfaction With Life (Diener et al., 1985), contendo cinco itens. Na presente amostra, o alfa de Cronbach foi de 0,84, o que define um bom nível de confiabilidade da escala (Pasquali, 2001).

Escala de Afetos Positivos e Negativos (Damásio et al., 2013): Esta escala é derivada da Positive and Negative Affect Scale for Children - PANAS-C8 e da ANAS-C34. A escala reduzida PANASC8 contém uma estrutura bifatorial com quatro itens por fator, totalizando oito itens, a fim de mensurar os afetos positivos e negativos. Na amostra do presente estudo, o alfa de Cronbach foi de 0,86 para afetos positivos e 0,73 para afetos negativos, considerados índices satisfatórios.

Escala de Coping Religioso-Espiritual (CRE) (Panzini, 2004). Foi desenvolvida por Pargament et al. (2000), adaptada e validada no contexto brasileiro por Panzini (2004). Esta escala avalia aspectos positivos e negativos do uso da religião/espiritualidade para manejo do estresse, tornando-se um instrumento compreensivo e útil a várias áreas da pesquisa científica. A CRE é composta por 87 itens subdivididos em oito fatores positivos (66 itens), que compõem a dimensão CRE Positivo (CREP) e quatro fatores negativos (21 itens), que compõem a dimensão CRE Negativo (CREN), de acordo com a classificação do enfrentamento. No presente estudo, o alfa de Cronbach foi de 0,96 , o que evidencia um nível alto de confiabilidade do instrumento.

Questionário Geral do Participante (Dados demográficos, econômicos e religiosos): desenvolvido pelos autores desta pesquisa, com o objetivo de identificar os participantes e suas condições socioeconômicas e demográficas, bem como aspectos relacionados ao casamento e ao envolvimento religioso.

\section{Procedimento}

Coleta de dados. Os participantes foram recrutados a partir da divulgação da pesquisa nas redes sociais dos pesquisadores, juntamente com o link de acesso do site de coletas de dados SurveyMonkey®. Ao acessar o link os participantes tiveram contato com uma breve apresentação da pesquisa e, em seguida, atestaram sua anuência por meio do Termo de Consentimento Livre e Esclarecido, sendo direcionados para o preenchimento das escalas.

Análises dos dados. Todos os dados foram organizados em um banco de dados e as análises ocorreram no software Statistical Package for the Social Sciences (SPSS, versão 22.0). Foi realizada análise descritiva (médias, medianas, desvios-padrão e porcentagens) dos escores obtidos por meio da aplicação das escalas, para caracterizar a amostradas. Para a realização das correlações aplicou-se o teste de normalidade Kolmogorov-Smirnov, sendo empregada estatística não-paramétrica para análise de correlações. Foram calculadas as correlações entre as medidas das variáveis contínuas a 


\section{CASAMENTOS DE LONGA DURAÇÃO}

partir do coeficiente de correlação de Spearman. O nível de significância adotado foi de $p \leq 0,05$. A força da grandeza do coeficiente de correlação foi avaliada conforme procedimento proposto por Zou et al. (2003), que consideram os valores de correlação próximos de 0,20 fracos, entre 0,30 e 0,50 moderados e acima de 0,50 fortes.

\section{RESULTADOS}

Em termos da caracterização da amostra, observou-se que 97,3\% acreditam em Deus. Destes, 41,4\% são católicos e 14,4\% se consideram sem religião, mas espiritualizados. Dos 2,7\% que não acreditam em Deus, $1,8 \%$ são agnósticos e $0,9 \%$ ateus. Considerando a frequência que os participantes estão presentes em suas instituições religiosas de referência (igreja, culto, centro, templo, ou outro encontro de natureza religiosa), 26,1\% vão semanal e esporadicamente e 0,9\% vão, mas não sabem definir. Questionados se a R/E repercute de forma positiva no casamento, 89,2\% responderam sim e 10,8\% disseram não. Acerca de quão importante tem sido a $\mathrm{R} / \mathrm{E}$ para lidar com fatores relacionados à vida conjugal, $53,2 \%$ disseram ser muito importante.

No que concerne à classificação dos níveis de Satisfação Conjugal $(M=29,05, D P=11,36)$ e considerando que menores índices na escala correspondem à maior qualidade conjugal, os resultados indicam que $41,4 \%$ da amostra apresentaram baixa pontuação na escala, referindo um nível de satisfação entre "bom" e "muito bom", e 18,9\% estão "acima da média". Como dito anteriormente, maiores pontuações na escala correspondem à existência de problemas conjugais. Assim, 27\% da amostra tiveram seu nível de satisfação entre "pobre" e "ruim", e 12,6\% apresentam "problemas severos" e "problemas muito severos". Apesar das mulheres da amostra apresentarem pontuações acima da obtida pelos homens em relação à satisfação conjugal, e considerando que houve nesse estudo maior participação feminina do que masculina, não foram observadas diferenças de gênero estatisticamente significativas entre as classificações da escala GRIMS com a variável sexo, o que corrobora com os resultados encontrados em estudos nacionais anteriores (Norgren et al., 2004; Rizzon et al., 2013).

Em relação ao Bem-estar Espiritual $(M=101,66 ; D P=15,06)$ e considerando a pontuação total da escala, tem-se que $66,7 \%$ da amostra apresentaram alto índice de bem-estar espiritual e $37 \%$ índice moderado ou baixo. A partir das médias das subescalas do bem-estar espiritual, percebe-se que a espiritualidade não se restringiu ao bem-estar religioso, representado por $69,0 \%$ dos sujeitos $(M=$ $51,89 ; D P=9,64)$, mas também foi significativa no bem-estar existencial com o índice de 52,2\% ( $M$ $=49,77 ; D P=6,95)$, sendo importante destacar que ambos escores das subscalas são considerados altos de acordo com a literatura (Silva, Souza, Nogueira, Moreira, \& Chaves, 2013). Esses resultados evidenciam que os participantes relacionam o bem-estar espiritual mais a uma referência a Deus do que à sensação de encontro de algo significativo na vida (Marques et al., 2009).

Em termos do Bem-estar Subjetivo, abordado neste estudo por meio da Escala de Satisfação de Vida $(M=18,08 ; D P=3,78)$ e Afetos Positivos e Negativos $(M=13,58 ; D P=2,96)$, observa-se que grande parte da amostra se mostrou um pouco insatisfeita com a vida $(45,9 \%), 27 \%$ pouco satisfeita, e 9,9\% neutro, ou seja, não estão satisfeitos nem insatisfeitos. Em relação ao gênero, de forma geral, os homens se mostram mais insatisfeitos com a vida do que as mulheres. A Satisfação de Vida, por envolver um julgamento global, também abarca os relacionamentos afetivos. Acerca dos escores obtidos da Escala de Afetos Positivos e Negativos, observou-se que a média dos afetos positivos ( $M$ $=13,59 ; D P=0,28)$ foi maior que a de afetos negativos $(M=7,77 ; D P=0,26)$. Dessa forma, podese observar que os participantes têm experienciado mais emoções e sentimentos positivos do que negativos. Os homens e as mulheres deste estudo avaliam de forma similar os afetos positivos e negativos. 
Neste estudo, a avaliação do Coping Religioso-Espiritual (CRE) foi realizada por meio do CRETOTAL, por ser o índice que apresenta o panorama conjunto da quantidade de CRE praticado pelo participante. E pela razão CREN/CREP, que revela a porcentagem de CRE Negativo em relação ao total de CRE Positivo. Desse modo, tem-se que a média de coping religioso-espiritual (CRETOTAL) praticado pelos participantes de estudo foi de $M=3,80(D P=0,39)$. Este valor é considerado alto, apontando que os sujeitos utilizam de forma significativa a religião e a espiritualidade para lidar com o estresse e adversidades em suas vidas (Borges, Santos, \& Pinheiro, 2015; Panzini, 2004). Em relação à Razão CREN/CREP, a média foi de $M=0,51(D P=0,17)$. Este índice é considerado baixo, tendo como parâmetro os valores de 0,20 a 5 , estipulados pelo estudo original (Panzini, 2004), apontado que a amostra deste estudo utiliza mais o CRE Positivo em relação ao CRE Negativo, indicando que as pessoas se voltam para a $\mathrm{R} / \mathrm{E}$ de forma positiva.

A partir dos resultados obtidos, foram examinadas as correlações entre os fatores dos construtos avaliados. O Quadro 1 resume as correlações encontradas pela associação dos domínios elencados no presente estudo.

Quadro 1. Matriz de correlações.

\begin{tabular}{ccccccc}
\hline & $\begin{array}{c}\text { Satisfação de } \\
\text { Vida }\end{array}$ & $\begin{array}{c}\text { Afetos } \\
\text { Positivos }\end{array}$ & $\begin{array}{c}\text { Afetos } \\
\text { Negativos }\end{array}$ & $\begin{array}{c}\text { Satisfação } \\
\text { Conjugal }\end{array}$ & $\begin{array}{c}\text { Bem-estar } \\
\text { Espiritual }\end{array}$ & $\begin{array}{c}\text { Coping religioso- } \\
\text { espiritual Total }\end{array}$ \\
\hline Satisfação de Vida & - & $0,48^{* *}$ & $-0,47^{* *}$ & $-0,59^{* *}$ & $0,33^{* *}$ & $0,21^{*}$ \\
Afetos Positivos & & - & $-0,51^{* *}$ & $-0,41^{* *}$ & $0,29^{* *}$ & $0,29^{* *}$ \\
Afetos Negativos & & - & - & $0,45^{* *}$ & $-0,24^{* *}$ & $-0,13$ \\
Satisfação Conjugal & & & & - & $-0,35^{* *}$ & $-0,18$ \\
Bem-estar Espiritual & & & & & - & $0,64^{* *}$ \\
Coping religioso- & & & & & & - \\
espiritual total & & & & & \\
\hline
\end{tabular}

Nota: ${ }^{*} p \leq 0.005 ; * * \leq 0.01$

Primeiramente podemos perceber que a amostra apresenta um elevado Bem-estar Subjetivo, uma vez que envolve altos níveis de afetos positivos e satisfação de vida e baixos índices de afetos negativos (Layous \& Zanon, 2014; Scorsolini-Comin et al., 2016). Esses achados são semelhantes aos descritos por Damásio et al. (2013) e Zanon et al. (2013).

Em termos das medidas da Satisfação Conjugal, é importante entender que a escala avalia os problemas conjugais. Partindo desse ponto temos que esta se correlacionou negativa e significativamente com o Bem-estar Espiritual ( $r h o=-0,39 ; p \leq 0,01)$, a Satisfação de Vida (rho = $0,55 ; p \leq 0,01)$ e com os Afetos Positivos ( $r h o=-0,42 ; p \leq 0,01)$. O nível das correlações negativas apresentadas é considerado moderado (Zou et al., 2003). No que tange à Satisfação Conjugal e Coping religioso-espiritual Total temos que, apesar de estarem significativamente correlacionadas ( $r h o=$ $0,18 ; p \leq 0,05)$, a força de grandeza nessa associação está entre fraca e nula, segundo os níveis estabelecidos por Zou et al. (2003). Esse resultado não nos permite assegurar a direção dessa relação, com pouca capacidade para explicar o fenômeno, devendo esse achado ser analisado com parcimônia. Acerca da Satisfação Conjugal e dos Afetos Negativos, encontrou-se uma correlação positiva de rho $=0,45(p \leq 0,01)$, considerada moderada.

Assim, quanto mais problemas conjugais, menor a satisfação conjugal, Bem-estar Espiritual e Bem-estar Subjetivo. Como a amostra apresentou baixos índices na escala, evidenciando maior qualidade conjugal, observamos que quanto mais Satisfação Conjugal a pessoa apresentar, menores serão os problemas conjugais e maior será seu Bem-estar Espiritual, ou seja, terão mais comunhão e relação íntima com Deus ou com algo que se considere uma força superior (considerando que a amostra apresentou maior escore para bem-estar religioso), bem como sua percepção em relação ao propósito da vida independentemente de uma referência religiosa (Marques et al., 2009). Outra 
observação é que quanto mais o indivíduo se sentir satisfeito em seu casamento maior será sua Satisfação de vida e seus afetos Positivos, podendo utilizar, ou não, da sua R/E para lidar com as adversidades do casamento. A relação positiva com os Afetos Negativos evidencia que quanto maiores os problemas conjugais, mais afetos negativos a pessoa manifestará, e quanto menos problemas conjugais, mais satisfação conjugal e menor manifestação de afetos negativos.

Houve correlação positiva forte $(r h o=0,63)$, de acordo com Zou et al. (2003) e significativa ( $p \leq$ 0,01) entre os resultados de Bem-estar Espiritual Total e Coping religioso-espiritual Total. Estes também se correlacionaram positivamente com os Afetos Positivos e a Satisfação de Vida, dois fatores que compõem o bem-estar subjetivo. Assim, podemos inferir que quanto mais a pessoa se relaciona com Deus, ou com algo que considera sagrado, experienciando o bem-estar de encontrar um propósito para a vida, mais ela se volta para a religião e utiliza da fé para lidar com o estresse e a resolução de problemas, repercutindo na avaliação positiva da vida (Albuquerque \& Tróccoli, 2004; Panzini \& Bandeira, 2005; Scorsolini-Comin et al., 2012).

No que se refere à duração do casamento, correlacionamos esse fator com os demais índices, obtendo os resultados apresentados no Quadro 2. O nível de significância adotado também foi de $p \leq$ 0,05 .

Quadro 2. Matriz de correlação em relação ao tempo de casamento/união estável.

\begin{tabular}{ccccccc}
\hline & $\begin{array}{c}\text { Satisfação } \\
\text { Conjugal }\end{array}$ & $\begin{array}{c}\text { Bem-estar } \\
\text { Espiritual }\end{array}$ & $\begin{array}{c}\text { Satisfação } \\
\text { de Vida }\end{array}$ & $\begin{array}{c}\text { Afetos } \\
\text { Positivos }\end{array}$ & $\begin{array}{c}\text { Afetos } \\
\text { Negativos }\end{array}$ & $\begin{array}{c}\text { Coping } \\
\text { religioso- } \\
\text { espiritual }\end{array}$ \\
\hline $\begin{array}{c}\text { Tempo de } \\
\text { casamento/união estável }\end{array}$ & $0,11(0,25)$ & $0,16(0,85)$ & $0,12(0,18)$ & $0,13(0,17)$ & $0,66(0,49)$ & $0,24 *(0,01)$ \\
\hline
\end{tabular}

Nota: ${ }^{*} p \leq 0.005$

Dessa maneira, observamos que embora a variável tempo de casamento/união estável apresente correlação significativa apenas com o Coping religioso-espiritual Total, ainda assim trata-se de uma associação fraca (Zou et al., 2003), com pouca capacidade de explicação do fenômeno. Essa baixa magnitude indica que esse esse achado ser analisado com parcimônia.

Retomando o objetivo do estudo, encontramos na amostra investigada correlação positiva significativa entre as variáveis Bem-estar Espiritual, Bem-estar Subjetivo (por meio dos índices apresentados entre Satisfação de Vida, Afetos Positivos e Afetos Negativos) e Coping religiosoespiritual. No que concerne às associações envolvendo as variáveis Satisfação Conjugal e Afetos Positivos, observamos que estão correlacionadas de maneira positiva, significativa e moderada entre si. Ambas apresentam correlações negativas significativas com os demais fatores, mas com ressalva para a correlação com o Coping religioso-espiritual Total, que não foi significativa e a grandeza entre fraca e nula (Zou et al., 2003), evidenciando pouco poder de explicação desse fenômeno. Consideramos também que a variável tempo de casamento/união não é significativa para a explicação dos demais fatores.

\section{DISCUSSÃO}

A Psicologia Positiva, sobretudo em sua primeira onda de estudos, tem se dedicado aos aspectos adaptativos dos indivíduos, com destaque para os elementos que podem ser promotores de bem-estar e que devem, portanto, ser potencializados no campo das intervenções em saúde (Campos et al., 2017; Giacomoni \& Scorsolini-Comin, 2020; Proyer et al., 2015; Reppold et al., 2015). Dentre os fatores abordados pela Psicologia Positiva podemos destacar o bem-estar subjetivo, as estratégias de enfrentamento, os afetos positivos e negativos, a satisfação de vida e a R/E, por exemplo, bem como 
o casamento como instituição positiva quando este é capaz de reforçar a autonomia e a intimidade dos indivíduos por meio das relações duradouras e de apoio. Essas dimensões estão presentes neste estudo, permitindo-nos compreender como as mesmas se relacionam no contexto da conjugalidade longeva.

Pelos dados da amostra, observa-se que a quantidade de pessoas que acreditam em Deus ou em um ser superior é expressiva, sendo composta em sua maioria por católicos, o que também é observado nos estudos de Alves-Silva el al. (2017), Estrada (2009) e Norgren et al. (2004), e que grande parte frequenta semanalmente as reuniões de suas denominações. A maioria dos participantes indica que a R/E repercute de forma positiva no casamento, sendo muito importante para lidar com fatores relacionados à vida conjugal como, por exemplo, a comunicação, a confiança, o respeito e o manejo de dificuldades diversas. Dessa forma, pode-se considerar que um número expressivo de participantes, além de acreditaram em algo que transcende a vida humana, participam de eventos religiosos e reconhecem que essa experiência está atrelada à forma como vivenciam seus relacionamentos conjugais.

Podemos inferir que a R/E está presente em casamentos longevos, sendo significativa na união. Esses achados são corroborados pelo estudo de Marks (2005) que identificou, por meio de suas investigações, que a fé, as crenças espirituais e as práticas religiosas influenciam a forma como as pessoas lidam com seus relacionamentos conjugais. E com as pesquisas de Mullins (2016) com casais longevos e David e Stafford (2013), ambas no contexto norteamericano, ao identificarem que a R/E está ligada à qualidade e estabilidade conjugal durante os anos, proporcionando apoio e reforçando a percepção dos casais de seus casamentos como abençoados e duradouros.

A presença da R/E nos casamentos longevos também pode ser percebida nos resultados de Coping religioso-espiritual Total e Bem-estar Espiritual Total, que apresentaram correlação positiva forte $(r h o=0,63)$, de acordo com Zou et al. (2003) e significativa $(p \leq 0,01)$, e da maior utilização do Coping religioso-espiritual Positivo em relação ao Coping religioso-espiritual Negativo. Por meio disso, entendemos que os participantes experimentam sensação de bem-estar ao se relacionarem com Deus e utilizam positivamente a R/E para encontrarem estratégias de enfrentamento das situações adversas que ocorrem em diferentes áreas da vida, incluindo a conjugalidade. Essa relação que se estabelece entre o sujeito e Deus e que repercute na conjugalidade pode ser compreendia a partir da Psicologia Positiva ao elucidar que o relacionamento com o sagrado aprimora um entendimento profundo da própria $\mathrm{R} / \mathrm{E}$, promovendo bem-estar, saúde mental, conhecimento da própria vida, recursos de enfrentamento de adversidade e bom funcionamento conjugal (Snyder \& Lopez, 2009).

Observou-se também que o Coping religioso-espiritual Total e Bem-Estar Espiritual Total se correlacionaram positivamente com o Bem-estar Subjetivo, representado pela Satisfação de Vida e Afetos Positivos. É importante destacar que neste estudo a dimensão Bem-estar Subjetivo foi avaliado por duas escalas diferentes (Escala de Satisfação de Vida e Escala de Afetos Positivos e Negativos), que fazem referência aos seus fatores constituintes. Os resultados obtidos das correlações entre esses fatores evidenciaram que a amostra apresenta um elevado Bem-estar Subjetivo (Albuquerque \& Tróccoli, 2004; Layous \& Zanon, 2014; Scorsolini-Comin et al., 2016). Assim, quanto mais a pessoa se relaciona com Deus, experienciando o bem-estar de encontrar um propósito para a vida e utiliza da R/E para lidar com fatores estressantes maiores serão seu bem-estar individual e as emoções positivas no casamento (Albuquerque \& Tróccoli, 2004; Panzini \& Bandeira, 2005; Scorsolini-Comin et al., 2012).

Em relação ao Bem-estar Subjetivo, pode-se dizer que esta noção está significativamente associada à de Satisfação Conjugal. Esses achados são corroborados por estudo anterior (Scorsolini-Comin \& Santos, 2012) que reportou uma associação positiva entre estar satisfeito no casamento e manifestar elevada satisfação de vida. A literatura aponta que pessoas casadas tendem a ser mais felizes pelo fato do relacionamento proporcionar maior intimidade por meio de relações duradouras e de apoio, reforçando a autoestima do indivíduo e gerando sensação de segurança na relação (Scorsolini-Comin et al., 2016). 
Enquanto medida de problemas conjugais, há que se considerar que os fatores do Bem-estar Subjetivo, da Satisfação de Vida e dos Afetos Positivos correlacionaram-se negativamente com a Satisfação Conjugal (rho $=-0,55 ; p \leq 0,01$ e rho $=-0,42 ; p \leq 0,01$, respectivamente). Por outro lado, o fator Afetos Negativos correlacionou-se positivamente com a Satisfação Conjugal ( $r h o=0,45$ ( $p \leq$ $0,01)$. Desse modo, percebemos que o casamento pode funcionar como locus de satisfação, qualidade conjugal e promoção de afetos positivos.

Esses resultados corroboram a literatura que tem apontado para uma realidade em que a satisfação conjugal encontra-se associada à qualidade e satisfação de vida e afetos positivos, indicando que pessoas casadas, principalmente em longa duração, são mais felizes e satisfeitas do que viúvos, divorciados e solteiros (Margelisch et al., 2015; Scorsolini-Comin \& Santos, 2012; Seligman, 2011; Snyder \& Lopes, 2009; Suhail \& Chaudhry, 2004). Dentre essas pesquisas podemos destacar a realizada por Viera (2005), ao encontrar em suas análises que a satisfação conjugal é preditora da satisfação de vida, mais do que qualquer outro aspecto da vida, por exemplo, emprego ou trabalho. Neste estudo, assim, como na pesquisa realizada por Scorsolini-Comin e Santos (2016) e Carr et al. (2014), os resultados mostraram que houve correlação positiva e significativa entre Satisfação Conjugal e Satisfação de Vida, ou seja, quanto maior o nível de bem-estar da pessoa casada, maior é sua satisfação com o seu relacionamento conjugal.

Podemos considerar que $27 \%$ da amostra tiveram seu nível de satisfação conjugal entre "pobre" e "ruim", e 12,6\% apresentaram "problemas severos" e "problemas muito severos", o que nos permite ponderar que as experiências pessoais consideradas negativas e desadaptativas, assim como a insatisfação, também podem compor a conjugalidade. Possivelmente nesse grupo existam mais problemas conjugais que podem estar diretamente relacionados com a satisfação, comunicação, interesse compartilhados, confiança e respeito. Além disso, esse achado reforça que a qualidade não é relacionada exclusivamente à estabilidade, uma vez que esses indivíduos vivenciam casamentos longevos, mas não de forma satisfatória. A estabilidade é definida por outras variáveis que podem ser contextuais e familiares, como a presença de filhos, por exemplo. Esse resultado também é corroborado pela variável tempo de casamento ter apresentado correlação positiva e significativa somente com o coping religioso/espiritual.

Em termos das medidas da Satisfação Conjugal, temos que esta se correlacionou negativa e significativamente com o Bem-estar Espiritual (rho $=-0,39 ; p \leq 0,01$ ), ou seja, quanto menos problemas conjugais e mais satisfação conjugal, mais Bem-estar Espiritual. Esses achados corroboram com os estudos de Norgren et al. (2004) e Mullins (2010) sobre a satisfação conjugal em casamentos de longa duração ao ressaltarem que níveis mais elevados de $\mathrm{R} / \mathrm{E}$ têm sido associados a níveis mais elevados de satisfação no casamento. Desse modo, observamos que quando as pessoas estão satisfeitas com seus casamentos buscam na R/E uma direção para lidar com possíveis demandas advindas da relação, que podem ser redução da expressão de afeto, coesão, proximidade, capacidade de resolução de problemas e habilidade de comunicação (Norgren et al., 2004). Como neste presente estudo o Bem-estar Espiritual Total está correlacionado com o Bem-estar Subjetivo, podemos supor que a procura pelo bem-estar espiritual está atrelado à maior manifestação da satisfação no casamento, encontrando em Deus uma maneira de reafirmar ainda mais o laço conjugal e uma forma de encontrar satisfação com a própria vida, experienciando afetos positivos.

Ainda com base nas associações deste estudo, o Bem-estar Espiritual Total e o Coping religiosoespiritual Total estiveram positiva e significativamente associados ao de Bem-estar Subjetivo, sugerindo proximidade entre conceitos e uma implicação direta da $\mathrm{R} / \mathrm{E}$ na manifestação do bem-estar. A forte associação entre Bem-estar Espiritual Total e Coping religioso-espiritual Total também revelou proximidade entre essas dimensões, indicando-as como índices importantes no estudo da R/E.

De acordo com os dados deste estudo, podemos concluir que a $\mathrm{R} / \mathrm{E}$ está presente em casamentos de longa duração, haja vista o elevado índice de bem-estar espiritual na amostra. Compreendendo, neste estudo, a R/E a partir das noções de Bem-estar Espiritual e Coping religioso-espiritual, podemos associar positivamente a satisfação conjugal à $\mathrm{R} / \mathrm{E}$. Assim, uma maior $\mathrm{R} / \mathrm{E}$ parece se associar à www.sp-ps.pt 
satisfação no casamento. Pode-se pensar que os recursos da R/E são preditores para a satisfação, sendo potentes no sentido de contribuir com um maior sentido de vida e bem-estar em pessoas engajadas nos casamentos longevos ou mesmo contribuir para a manutenção do casamento. Apesar desse achado corroborar com as evidências aqui recuperadas, pondera-se sobre a necessidade de investigar melhor, nessa amostra e em outros grupos, os fatores preditores da satisfação conjugal, considerando o recorte específico dos casamentos de longa duração.

À guisa de conclusão, a hipótese inicial foi aceita, evidenciando que pessoas com maiores índices de $\mathrm{R} / \mathrm{E}$ tendem a manifestar maior satisfação no casamento e menores problemas conjugais, o que pode indicar que a $\mathrm{R} / \mathrm{E}$ faz parte do universo desses casais, favorecendo que permaneçam juntos. As correlações evidenciaram que essas dimensões impactam a qualidade conjugal e vice-versa, possibilitando compreender a intenção que se estabelece entre elas e a necessidades de todos os elementos estejam presentes para que a conjugalidade seja considerada satisfatória.

Por estar relacionada à manutenção do casamento, a $\mathrm{R} / \mathrm{E}$ deve ser melhor investigada em estudos vindouros, compreendendo melhor se a R/E constitui um elemento que pode indicar uma "fuga" das vivências conjugais consideradas desadaptativas ou algo que, de fato, possa dar sentido à relação para além do aspecto da satisfação, o que pode ser um aspecto típico em casamentos longevos. Assim, pode-se questionar, para além dos dados, se a R/E não obscureceria um maior investimento na própria relação, funcionado como um mecanismo de fuga no enfrentamento dos problemas que podem emergir em um casamento de longa duração. O suporte a essas considerações deve ser oferecido por delineamentos posteriores, investigando os preditores tanto da $\mathrm{R} / \mathrm{E}$ quanto da satisfação conjugal nesses participantes.

Como limitações deste estudo destaca-se que a coleta foi online, não sendo possível verificar se houve a participação de ambos os cônjuges, uma vez que não fazia parte dos critérios de inclusão a participação dos dois membros do casal. A análise diádica poderia oferecer suporte a outras considerações. Outra limitação na composição da amostra foi o fato de a maioria dos participantes ser do sexo feminino, sendo necessário ampliar a representatividade masculina, o que pode contribuir com novas análises a partir de marcadores de gênero.

O tempo de duração do casamento não se mostrou associado aos fatores de Bem-estar Espiritual, Satisfação de Vida, Afetos Positivos, Afetos Negativos, somente ao Coping religioso-espiritual. Destaca-se, então, a necessidade de estudos futuros que avaliem essas dimensões em casais (díades), o que pode contribuir para investigar se esses índices refletem a vivência do cônjuge ou da situação conjugal. Em estudos futuros, recomenda-se também a investigação de preditores da satisfação conjugal e da $\mathrm{R} / \mathrm{E}$ nesse público, adensando o repertório sobre os casais longevos e suas características. A compreensão dessas associações, no entanto, é um passo importante para o estabelecimento de delineamentos futuros, em uma busca pelo diálogo com a produção existente e a abertura para novos posicionamentos e inteligibilidades acerca do tema.

\section{REFERÊNCIAS}

Albuquerque, A. S., \& Tróccoli, B. T. (2004). Desenvolvimento de uma escala de bem-estar subjetivo. Psicologia: Teoria e Pesquisa, 22(2), 153-164. https://doi.org/10.1590/S010237722004000200008

Alves-Silva, J. D., Scorsolini-Comin, F., \& Santos, M. A. (2017). Bodas para uma vida: motivos para manter um casamento de longa duração. Temas em Psicologia (Ribeirão Preto), 25(2), 487-501. https://doi.org/10.9788/TP2017.2-05

Borges, M. S., Santos, M. B. C., \& Pinheiro, T. G. (2015). Representações sociais sobre religião e espiritualidade. Revista Brasileira de Enfermagem, 68(4), 609-16. https://doi.org/10.1590/00347167.2015680406i 
Campos, S. O., Scorsolini-Comin, F., \& Santos, M. A. (2017). Transformações da conjugalidade em casamentos de longa duração. Psicologia Clínica, 29(1), 69-89.

Cunha, V. F., Pillon, S. C., Zafar, S., Wagstaff, C., \& Scorsolini-Comin, F. (2020). Brazilian nurses' concept of religion, religiosity, and spirituality: a qualitative descriptive study. Journal of Nursing and Health Sciences, 22(4), 1161-1168. https://doi.org/10.1111/nhs.12788

Cunha, V. F., \& Scorsolini-Comin, F. (2020). Brazilian psychotherapists and the dimension of religiosity/spirituality. Counselling and Psychotherapy Research, 20(4), 1-9. https://doi.org/10.1002/capr.12357

Carr, D., Freedman, V. A., Cornman, J. C., \& Schwarz, N. (2014). Happy Marriage, Happy Life? Marital Quality and Subjective Well-Being in Later Life. Journal of Marriage and Family, 76(5), 930-948. https://doi.org/10.1111/jomf.12133

Costa, C. B., Falcke, C., \& Mosmann, C. P. (2015). Conflitos conjugais em casamentos de longa duração: motivos e sentimentos. Psicologia em Estudo, 20(3), 411-423. https://doi.org/10.4025/psicolestud.v20i3.27817

Costa, C. B., \& Mosmann, C. P. (2015). Relacionamentos conjugais na atualidade: Percepções de indivíduos em casamentos de longa duração. Revista da SPAGESP, 16(2), 16-31.

Damásio, B. F., Pacico, J. C., Poletto, M., \& Koller, S. H. (2013). Refinement and psychometric properties of the eight-item Brazilian Positive and Negative Affective Schedule for Children (PANAS-C8). Journal of Happiness Studies, 14(4), 1363-1378. https://doi.org/10.1007/s10902012-9383-x

David, P., \& Stafford, L. (2013). A Relational Approach to Religion and Spirituality in Marriage. The Role of Couples' Religious Communication in Marital Satisfaction. Journal of Family Issues, 36(2), 232-249. https://doi.org/10.1177/0192513X13485922

Diener, E. D., Emmons, R. A., Larsen, R. J., \& Griffin, S. (1985). The satisfaction with life scale. Journal of Personality Assessment, 49(1), 71-75. https://doi.org/10.1207/s15327752jpa4901_13

Estrada, R. I. (2009). An Examination of Love and Marital Satisfaction in Long-term Marriages. (Master Thesis). University of Denver, EUA.

Falcke, D. (2003). Águas passadas não movem moinhos: as experiências na família de origem como preditoras da qualidade do relacionamento conjugal. (Tese de Doutorado). Pontifícia Universidade Católica do Rio Grande do Sul, Porto Alegre, RS, Brasil.

Garcia, A., \& Maciel, M. G. (2008). A influência da religião na busca do futuro cônjuge: um estudo preliminar em comunidades evangélicas. Psicologia: Teoria e Prática, 10(1), 95-112.

Geografia e Estatística -IBGE. (2015). Estatística do Registro Civil. Rio de Janeiro.

Giacomoni, C. H., \& Scorsolini-Comin, F. (2020). Temas especiais em Psicologia Positiva. Vozes.

Goulart, S. A., Oliveira, A. C. G. A., Santos, M. A., \& Scorsolini-Comin, F. (2019). Fatores relacionados aos casamentos de longa duração: panorama a partir de uma revisão integrativa. Psico, 50, 30370. https://doi.org/10.15448/1980-8623.2019.2.30370

Grizólio, T. C., Santos, M. A., \& Scorsolini-Comin, F. (2020). Razões para a manutenção do laço conjugal diante de eventos críticos em casamentos longevos. Contextos Clínicos, 13(3), 762-785. https://doi.org/10.4013/ctc.2020.133.03

Koller, S. H., \& Hutz, C. (1996). Meninos e meninas em situação de rua: Dinâmica, diversidade e definição. Coletâneas da ANPEPP, 1, 11-34.

Layous, K., \& Zanon, C. (2014). Avaliação da felicidade subjetiva: para além dos dados de autorrelato. In C. S. Hutz (Org.). Avaliação em Psicologia Positiva (pp. 23-41). Artmed.

Margelisch, K., Schneewind, K. A., Violette, J., \& Perrig-Chiello, P. (2015). Marital stability, satisfaction and well-being in old age: variability and continuity in long-term continuously married older persons. Aging Mental Health, 21(4), 389-398. https://doi.org/10.1080/13607863.2015.1102197 
Marks, L. (2005). How Does Religion Influence Marriage? Christian, Jewish, Mormon, and Muslim Perspectives. Marriage \& Family Review, 38(1), 85-111. https://doi.org/10.1300/J002v38n01_07

Marques, L. F., Sarriera, J. C., \& Dell'Aglio, D. D. (2009). Adaptação e validação da escala de bemestar espiritual (EBE). Avaliação Psicológica, 8(2), 179-186.

Mosmann, C., Wagner, A., \& Féres-Carneiro, T. (2006). Qualidade conjugal: mapeando conceitos. Paidéia (Ribeirão Preto), 16(35), 315-325. https://doi.org/10.1590/S0103-863X2006000300003

Mullins, D. F. (2016). The effects of religion on enduring marriages. Social Scienses, 24(5), 1 - 14. https://doi.org/10.3390/socsci5020024

Nadal, A. R. C., Hardy, S. A., \& Barry, C. M. (2018). Understanding the roles of religiosity and spirituality in emerging adults in the united states. Psychology of Religion and Spirituality, 10(1), 30-43. https://doi.org/10.1037/rel0000104

Norgren, M. B., Souza, R. M., Kaslow, F., Hammerschmidt, H., \& Sharlin, S. A. (2004). Satisfação conjugal em casamentos de longa duração: uma construção possível. Estudos de Psicologia, 9(3), 575-584. https://doi.org/10.1590/S1413-294X2004000300020

Panzini, R. G. (2004). Escala de coping religioso-espiritual (Escala CRE): Tradução, adaptação e validação da escala Rcope, abordando relações com saúde e qualidade de vida. (Dissertação de Mestrado) Universidade Federal do Rio Grande do Sul. https://doi.org/10.1590/S141373722005000300019

Panzini, R. G., \& Bandeira, D. R. (2005). Escala de coping religioso-espiritual (Escala CRE): elaboração e validação de construto. Psicologia em Estudo, 10(3), 507-516. https://doi.org/10.1590/S1413-73722005000300019

Pargament, K. I., Koenig, H. G. \& Perez, L. M. (2000). The many methods of religious coping: Development and initial validation of the RCOPE. Journal of Clinical Psychology, 56(4), 519543. https://doi.org/10.1002/(SICI)1097-4679(200004)56:4<519::AID-JCLP6>3.0.CO;2-1

Pasquali, L. (Org.). (2001). Técnicas de exame psicológico: Manual. Casa do Psicólogo.

Proyer, R. T., Wellenzohn, S., Gander, F., \& Ruch, W. (2015). Toward a better understanding of what makes positive psychology interventions work: predicting happiness and depression from the person $\times$ intervention fit in a follow-up after 3.5 years. Applied Psychology: Health and WellBeing, 7, 108-128. https://doi.org/10.1111/aphw.12039

Rainal, P., \& Maity, T. (2018). An Empirical Study on Marital Satisfaction between Arranged and Self Marriage Couples in Bangalore. The International Journal of Indian Psychology, 6(1), 101108.

Reppold, C. T., Gurgel, L. G., \& Schiavon, C. C. (2015). Research in Positive Psychology: a systematic literature review. Psico-USF, 20(2), 275-285. https://doi.org/10.1590/14138271201520008

Rizzon, A. L. C., Mosmann, C. P., \& Wagner, A. (2013). A qualidade conjugal e os elementos do amor: um estudo correlacional. Contextos Clínicos, 6(1), 41-49. https://doi.org/10.4013/ctc.2013.61.05

Scorsolini-Comin, F., Fontaine, A. M. G. V., Barroso, S. M., \& Santos, M. A. (2015). Relações entre conjugalidade dos pais, conjugalidade dos filhos e bem-estar subjetivo. Psico-USF, 20(3), 481492. https://doi.org/10.1590/1413-82712015200310

Scorsolini-Comin, F., Fontaine, A. M. G. V., Barroso, S. M., \& Santos, M. A. (2016). Factors associated with the subjective well-being in married and unmarried people. Estudos de Psicologia, 33(2), 313-324. https://doi.org/10.1590/1982-02752016000200013

Scorsolini-Comin, F., \& Santos, M. A. (2011). Ajustamento diádico e satisfação conjugal: correlações entre os domínios de duas escalas de avaliação da conjugalidade. Psicologia: Reflexão e Crítica, 24(3), 439-447. https://doi.org/10.1590/S0102-79722011000400005 


\section{CASAMENTOS DE LONGA DURAÇÃO}

Scorsolini-Comin, F., \& Santos, M. A. (2012). Correlations between subjective well-being, Dyadic adjustment and marital satisfaction in Brazilian married people. The Spanish Journal of Psychology, 15(1), 166-176. https://doi.org/10.5209/rev_SJOP.2012.v15.n1.37304

Scorsolini-Comin, F., Santos, M. A., \& Souza, R. M. (2012). Expressão do afeto e bem-estar subjetivo em pessoas casadas. Estudos de Psicologia (Natal), 17(2), 321-328. https://doi.org/10.1590/S1413-294X2012000200017

Seligman, M. E. P. (2011). Florescer: uma nova compreensão sobre a natureza da felicidade e do bem-estar. Objetiva.

Silva, L. A., Scorsolini-Comin, F., \& Santos, M. A. (2017). Casamentos de longa duração: recursos pessoais como estratégias de manutenção do laço conjugal. Psico-USF, 22(2), 323-335. https://doi.org/10.1590/1413-82712017220211

Silva, R. P., Souza P., Nogueira D. A., Moreira D. S., \& Chaves, E. C. L. (2013). Relação entre bemestar espiritual, características sociodemográficas e consumo de álcool e outras drogas por estudantes. Jornal Brasileiro de Psiquiatria, 62(3), 191-198. https://doi.org/10.1590/S004720852013000300003

Snyder, C. R., \& Lopez, S. J. (2009). Psicologia Positiva: uma abordagem científica e práticas humanas. Artmed.

Suhail, K., \& Chaudhry, H. R. (2004). Predictors of subjective wellbeing in an Eastern Muslim culture. Journal of Social and Clinical Psychology, 23(3), 359-376. https://doi.org/10.1521/jscp.23.3.359.35451

Zanon, C., Bastianello, M. R., Pacico, J. C., \& Hutz, C. S. (2013). Desenvolvimento e validação de uma escala de afetos positivos e negativos. Psico-USF, 18(2), 193-201. https://doi.org/10.1590/S1413-82712013000200003

Zou, K. H., Tuncali, K., \& Silverman, S. G. (2003). Correlation and simple linear regression. Radiology, 227, 617-628. https://doi.org/10.1148/radiol.2273011499 